\title{
Dipolar Interactions and Protein \\ Hydration in Highly Concentrated \\ Antibody Formulations
}

Josef Hartl ${ }^{1}$, Sergej Friesen ${ }^{2}$, Diethelm Johannsmann ${ }^{3}$, Richard Buchner ${ }^{2}$, Dariush Hinderberger ${ }^{1}$, Michaela Blech ${ }^{4}$ and Patrick Garidel ${ }^{4 *}$

[1] Institute of Chemistry, Martin-Luther-University of Halle-Wittenberg, D-06120 Halle (Saale), Germany

[2] University of Regensburg, Institute of Physical and Theoretical Chemistry, University of Regensburg, D-93053 Regensburg, Germany

[3] Institute of Physical Chemistry, Clausthal University of Technology, D-38678 Clausthal-Zellerfeld, Germany

[4] Boehringer Ingelheim Pharma GmbH \& Co. KG, Innovation Unit, PDB,D-88397 Biberach an der Riss, Germany

\section{Supporting Information}

\section{Electronic Supplementary Information (ESI)}

\section{Corresponding Author}

*Patrick Garidel (Dr. rer. nat.), Boehringer Ingelheim Pharma GmbH \& Co. KG, Innovation Unit, PDB, Birkendorfer Strasse 65, 88397, Biberach an der Riss, Germany, E-mail: Patrick.Garidel@boehringeringelheim.com 


\section{A) Degradation and protein aggregation during dielectric relaxation spectroscopy experiments}

During dielectric relaxation spectroscopy (DRS) experiments, an alternating electric field is applied on proteinaceous samples. However, its impact for protein degradation and protein aggregation especially at protein concentrations $>100 \mathrm{mg} / \mathrm{ml}$ is unclear. In this section, we provide size exclusion chromatography data of monoclonal antibody solutions before and after DRS measurements to evaluate the DRS-induced protein degradation (low molecular weight species, LMW) and the potential formation of high molecular weight (HMW, aggregation) species.

\section{Size exclusion chromatography (SEC)}

SEC analyses were performed by using an Acquity UPLC H-class system (Waters, Milford, USA) with an injection volume of $6 \mu \mathrm{l}$ sample. UP-SEC analyses were performed at room temperature with a flow rate of $0.2 \mathrm{ml} \cdot \mathrm{min}^{-1}$ of a solution containing $200 \mathrm{mM} \mathrm{L}$-arginine, $120 \mathrm{mM}$ ammonium sulfate, $10 \%(\mathrm{v} / \mathrm{v})$ isopropanol, $32 \mathrm{mM}$ phosphoric acid, pH 7.3 as mobile phase, and using an UP-SEC column (Acquity UPLC ${ }^{\circ}$ BEH SEC 200Å, functional group: diol, length: $30 \mathrm{~cm}$, interior diameter: $4.6 \mathrm{~mm}$, pore size: 20 $\mathrm{nm}$, Waters) was used. Highly concentrated protein samples were diluted with the mobile phase to a protein concentration of $5 \mathrm{mg} / \mathrm{ml}$ to reduce the impact of formulation buffer and excipients on reversible protein association. Monomer, aggregate, and fragment contents were detected at $280 \mathrm{~nm}$ as a proportion of the total signal area of the chromatogram. Data are illustrated in Fig. S1 and Fig. S2. 


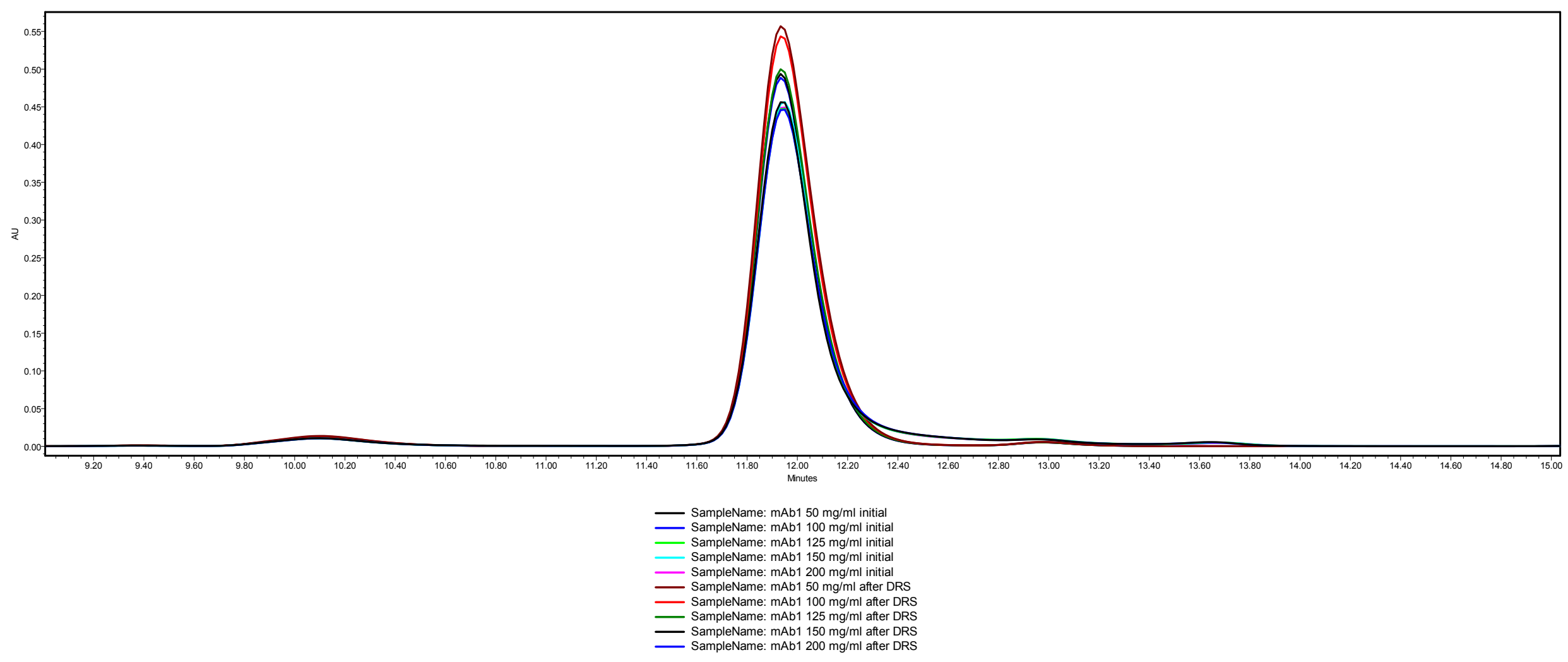

Figure S1: Size exclusion chromatographic (SEC) results of mAb1 in water at pH 6.0 at different concentration before (initial) and after dielectric relaxation measurements (DRS). A slight decrease in the monomer peak at 12 min was observed after DRS experiments. No relevant changes were observed prior and post DRS measurement for the formation of LMWs and HMWs. 
5

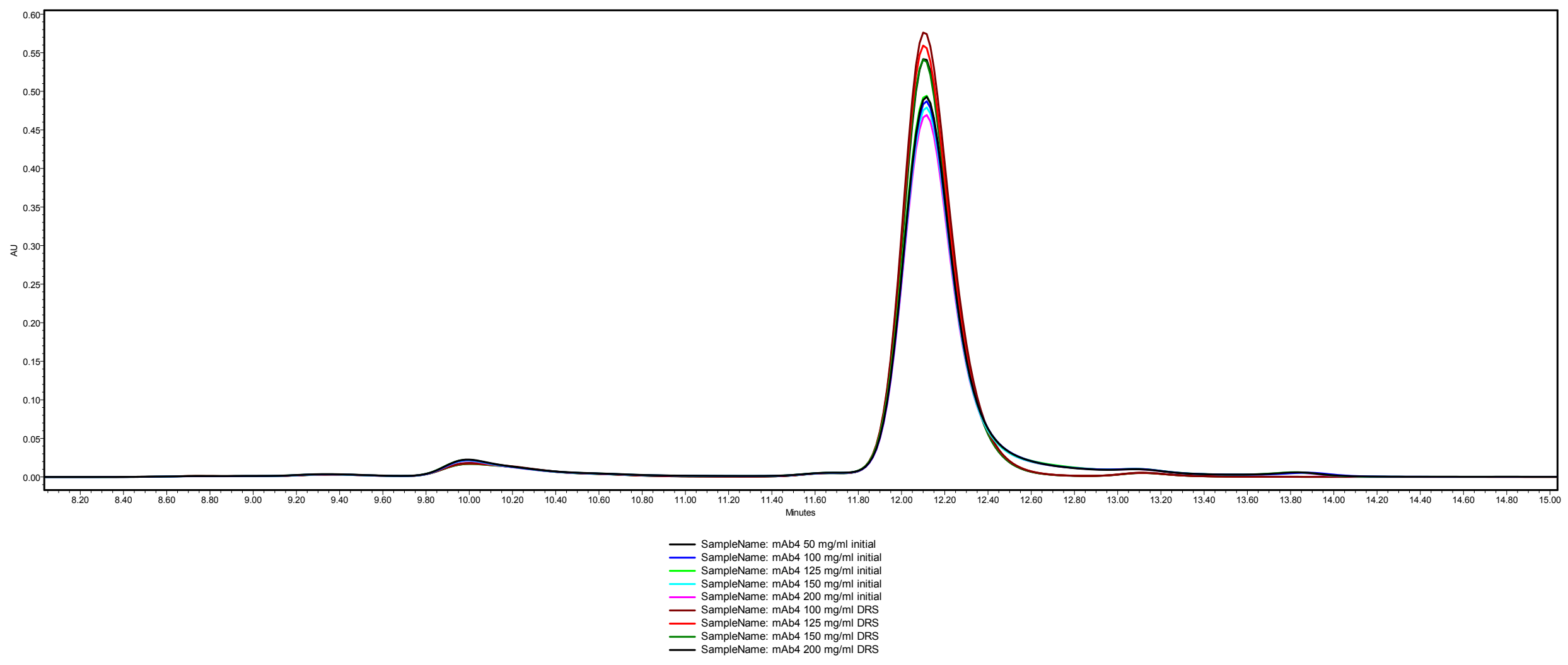

Figure S2: Size exclusion chromatographic (SEC) results of mAb4 in water at pH 6.0 at different concentration before (initial) and after dielectric relaxation measurements (DRS). A slight decrease in the monomer peak at $12 \mathrm{~min}$ was observed after DRS experiments. No relevant changes were observed prior and post DRS measurement for the formation of LOWs and HOWs. 


\section{B) Isoelectric points of protein molecules}

The isoelectric points (IPs) were calculated using the Molecular Operating Environment (MOE) software package (Chemical Computing Group Inc., Montreal, Canada). The individual amino acid pKa were estimated from 3D coordinates by PROPKA. These calculations take not into account the effect of chemical environment and charge shielding effects.

A ZetaPALS (Brookhaven Instruments Corporation, Holtsville, NY) was used to measure the electrophoretic mobility $\left(U_{E}\right)$ of the antibodies via phase analysis. Here the phase difference of through the sample - scattered light and not scattered light where compared. The difference of the time $(t)$ derivated phase $\left(d \Phi_{s}\right)$ is related to the electrophoretic mobility by

$$
\frac{d \Phi_{S}(t)}{d t}=q \cdot\left(v_{E} \pm v_{c}\right) \text { with } \quad v_{e}=U_{E} \cdot E(t)
$$

Where $\mathrm{q}$ is the momentum transfer vector, $v_{E}$ is the velocity of the particle in the field, $v_{c}$ is the fieldindependent velocity and $E(t)$ is the applied time-dependent electric field.

For validating the method the measurement parameters were optimized (applied electric field strength, reference factor, electrical voltage, number of cycles, number of runs, ideal sample concentration) and different measurement modes were tested (phase analysis compared to Doppler frequency shift). In our case we relied on the phase analysis light scattering (PALS) method because the phase different can be detected and quantified faster than the frequency shift. All measurements were done in the automatic field modus and voltage mode which usually set voltage between 4-8 V. The autocorrelation of $\Delta \Phi_{S}$ was set to 0.015 . Each sample was performed 10 times and temperature was set at $25{ }^{\circ} \mathrm{C}$. For the antibody formulations an RI of 1.333 was used. The calculation of the electrokinetic potential $(\zeta)$ out of the electrophoretic mobility was done automatically by the instrument-software.

$$
\zeta=\frac{3 U_{E} \eta}{2 \varepsilon_{0} \varepsilon_{r} f(\kappa a)}
$$

with $U_{E}$ the electrophoretic mobility, $\eta$ the viscosity of the sample, $\varepsilon_{0}$ the dielectric constant of vacuum, $\varepsilon_{r}$ the relative dielectric constant and $\mathrm{f}(\kappa \mathrm{k})$ the Henry's constant (see Wagner et al., Colloids and Surfaces A: Physicochem. Eng. Aspects 2012, 415, 421-430). 
The approximation of Henry's function was calculated out of the Hückel-Debye parameter $(\kappa)$ and the radius of the protein $\left(R_{p}\right)$, individually for each sample:

$$
f\left(\kappa R_{p}\right)=1+\frac{0.5}{1+\exp \left[a\left(1-\lg \left(\kappa R_{p}\right)\right)\right]}
$$

With $\mathrm{a}=2.8$ for $\kappa R_{p}<10$ and $\mathrm{a}=2.5$ for $\kappa R_{p}>10$.

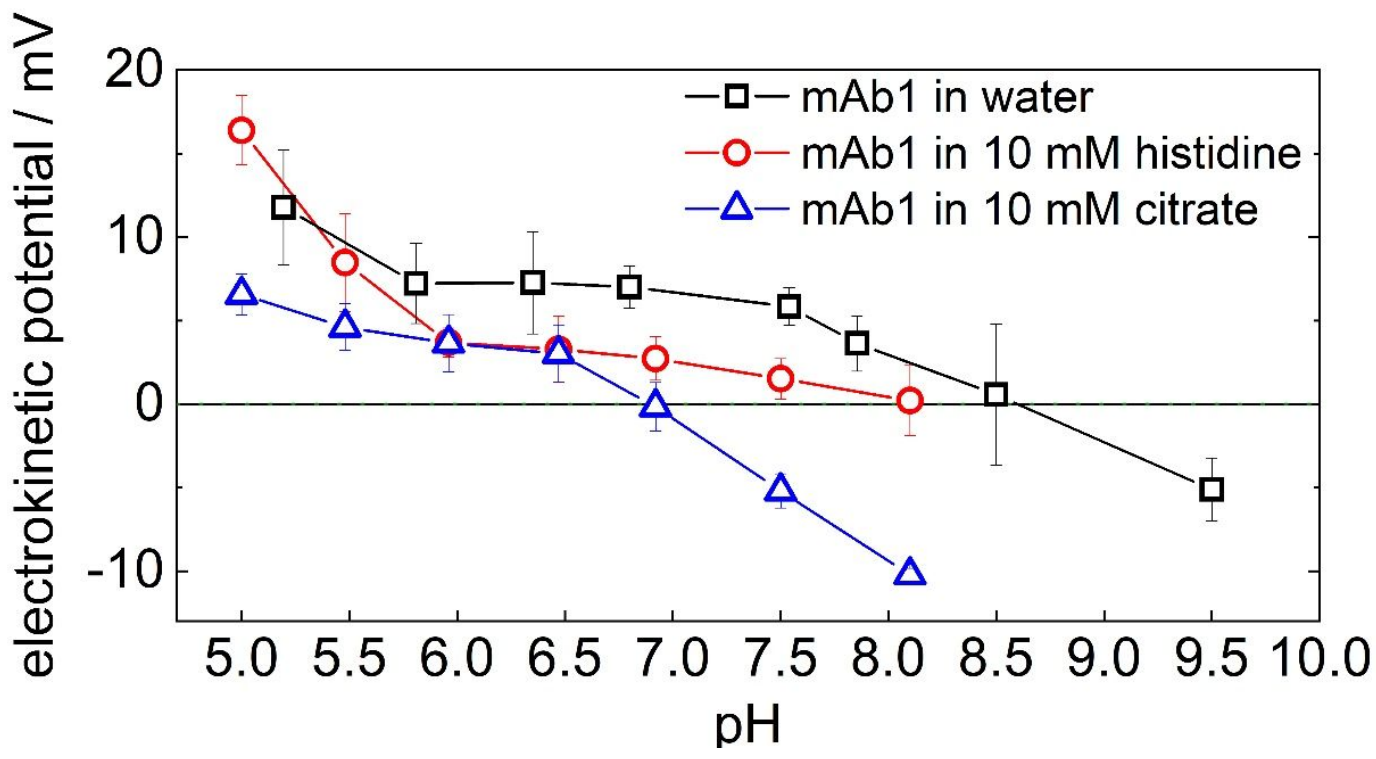

Figure S3: Electrokinetic potential vs. $\mathrm{pH}$ for $\mathrm{mAb1}$ in water, $10 \mathrm{mM}$ histidine and $10 \mathrm{mM}$ citrate. Chloride acts as counterion of $\mathrm{mAb1}$ in the water and in $10 \mathrm{mM}$ histidine samples. The mAb1 concentration was set to $10 \mathrm{mg} / \mathrm{ml}$. As a result of the decreasing surface charge, the electrokinetic potential decreases with more alkaline $\mathrm{pH}$. These effects are stronger in $10 \mathrm{mM}$ citrate compared to the chloride containing solutions. Shielding of $10 \mathrm{mM}$ citrate alters the isoelectric point of $\mathrm{mAb} 1$ from IEP $\approx 8.5$ to IEP $\approx 7.0$ 


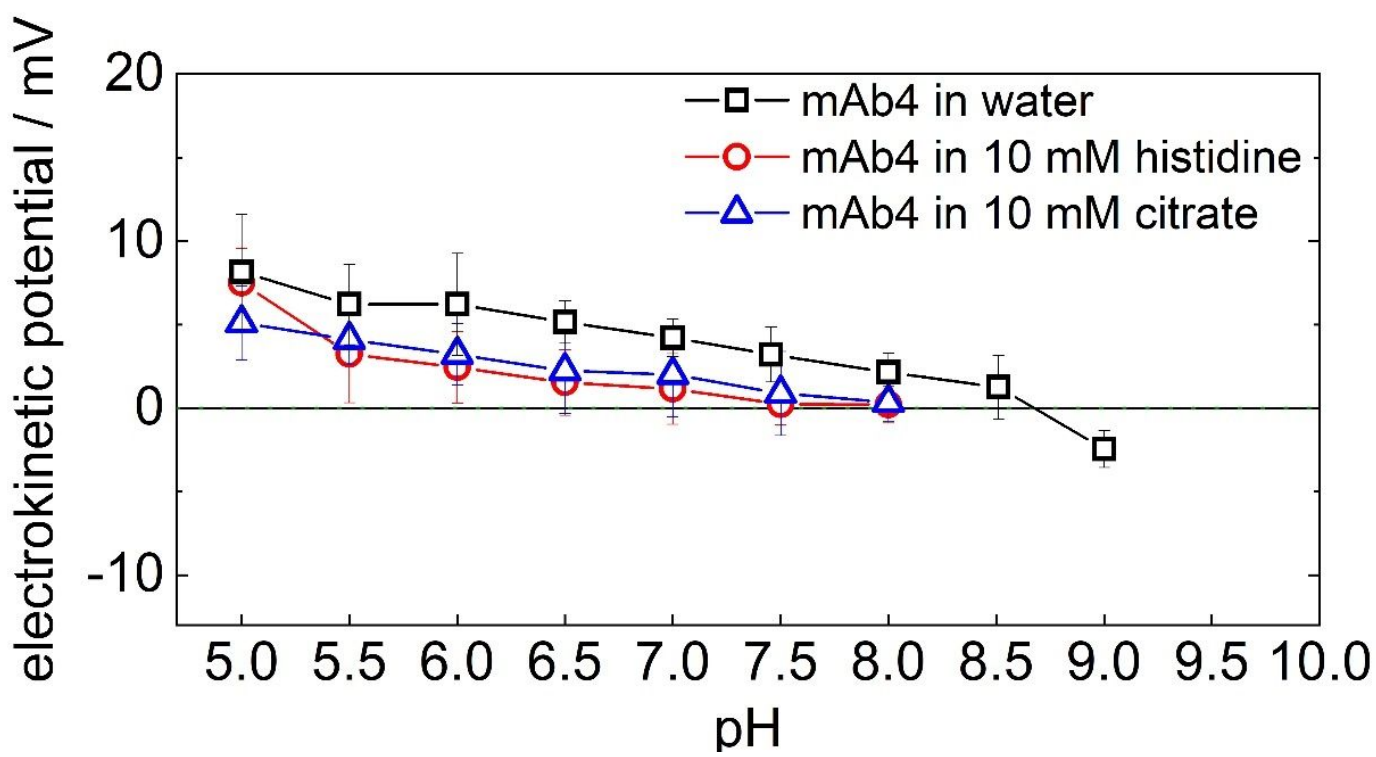

Figure S4: Electrokinetic potential vs. $\mathrm{pH}$ for mAb4 in water, $10 \mathrm{mM}$ histidine and $10 \mathrm{mM}$ citrate. Chloride acts as counterion of mAb4 in the water and in $10 \mathrm{mM}$ histidine samples. The mAb4 concentration was set to $10 \mathrm{mg} / \mathrm{ml}$. As a result of the decreasing surface charge, the electrokinetic potential decreases with more alkaline $\mathrm{pH}$. Compared to mAb1, the IEP of mAb4 does not change with citrate as counter ion. 
C) Second virial coefficient $A_{2}$ in different formulations.

Our study reports values for the second osmotic virial coefficient, $A_{2}$, as determined by multi-angle light scattering. Fig. $\mathrm{S} 5$ and Fig. $\mathrm{S} 6$ provides Zimm plots of mAb1 and mAb4.
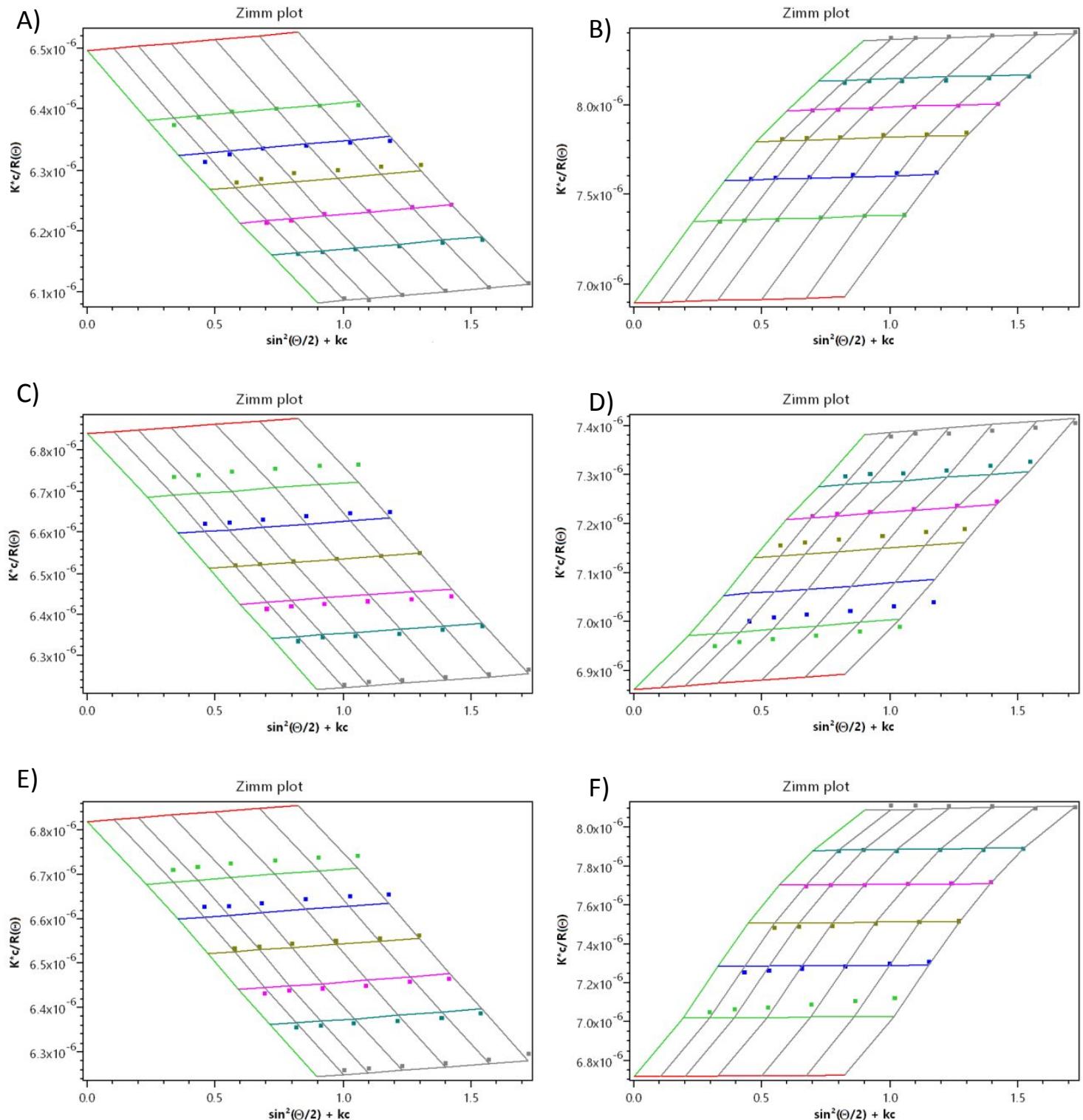

Figure S5: Zimm plots for MALS $A_{2}$ measurements of mAb1 Injection: 40, 60, 80, 100, 120 and $150 \mu \mathrm{l}$ of $10 \mathrm{mg} / \mathrm{ml} \mathrm{mAb1}$ in different buffers. A) mAb1 in $10 \mathrm{mM}$ citrate $\mathrm{pH} 5.0, \mathrm{~B}) \mathrm{mAb} 1$ in $10 \mathrm{mM}$ citrate $\mathrm{pH}$ 6.0, C) $\mathrm{mAb} 1$ in $10 \mathrm{mM}$ citrate $\mathrm{pH}$ 7.0, D) $\mathrm{mAb1}$ in $10 \mathrm{mM}$ histidine $\mathrm{pH}$ 5.0, E) mAb1 in $10 \mathrm{mM}$ histidine $\mathrm{pH}$ 6.0, F) mAb1 in $10 \mathrm{mM}$ histidine $\mathrm{pH} 7.0$. 

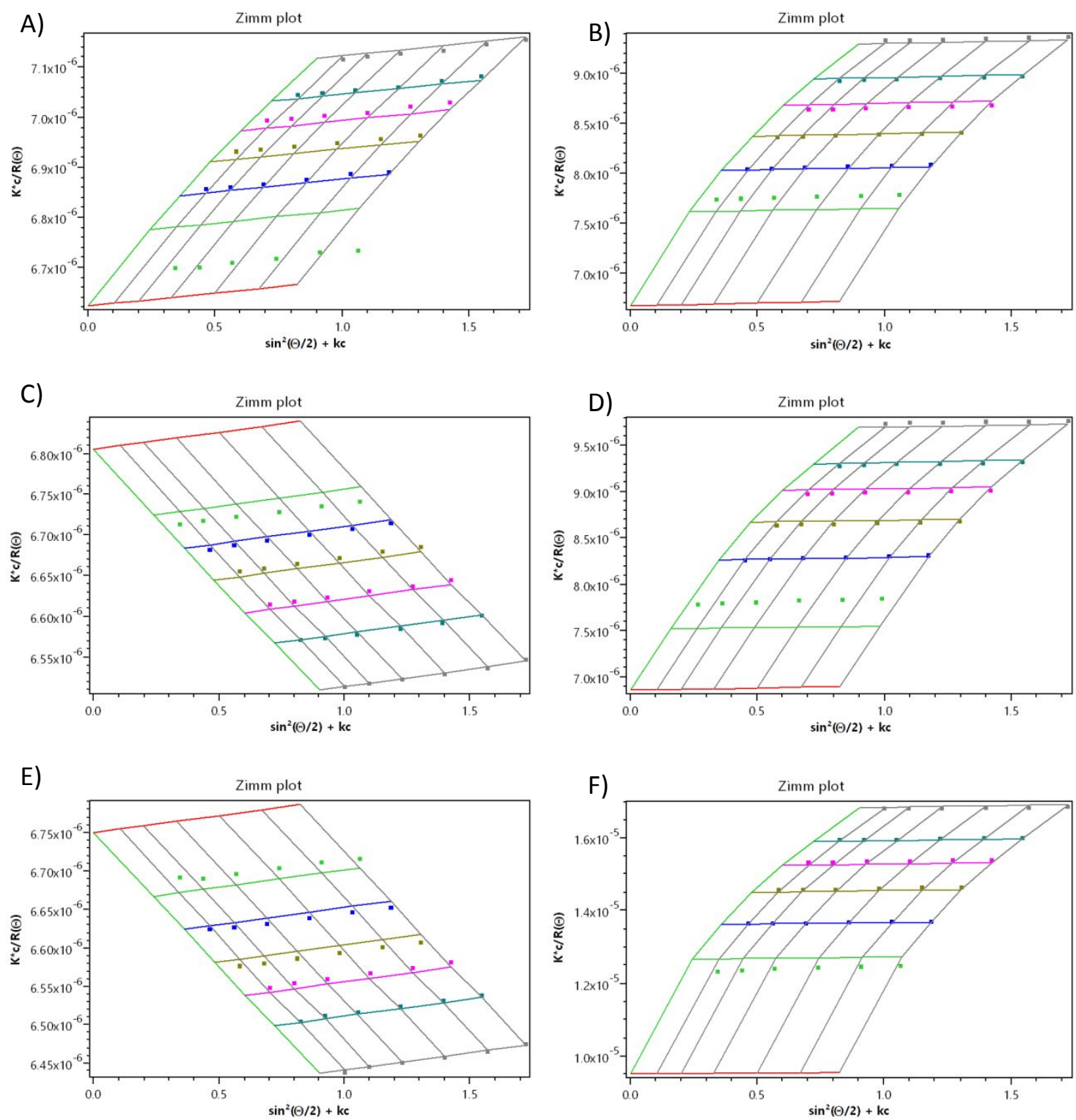

Figure S6: Zimm plots for MALS (Multi Angle Light Scattering) $A_{2}$ measurements of mAb4 Injection: 40, $60,80,100,120$ and $150 \mu \mathrm{l}$ of $10 \mathrm{mg} / \mathrm{ml} \mathrm{mAb4}$ in different buffers. A) mAb4 in $10 \mathrm{mM}$ citrate $\mathrm{pH}$ 5.0, B) mAb4 in $10 \mathrm{mM}$ citrate $\mathrm{pH} 6.0, \mathrm{C}$ ) mAb4 in $10 \mathrm{mM}$ citrate $\mathrm{pH} 7.0, \mathrm{D}) \mathrm{mAb} 4$ in $10 \mathrm{mM}$ histidine $\mathrm{pH}$ 5.0, E) mAb4 in $10 \mathrm{mM}$ histidine $\mathrm{pH} 6.0, \mathrm{~F}$ ) mAb4 in $10 \mathrm{mM}$ histidine $\mathrm{pH}$ 7.0. 


\section{D) Viscosity of mAbs in different formulations.}

As mentioned in our study, the reduced values of tau_2 $\left(\tau_{2}\right)$ are related to the reduced viscosity of the histidine containing solution. This argument has been presented and described qualitatively in the text. The discussed viscosity values are listed in Fig. S7.

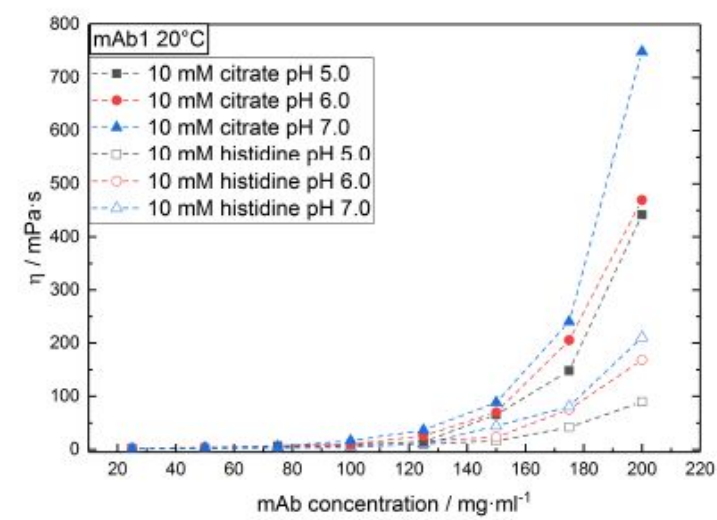

(a)

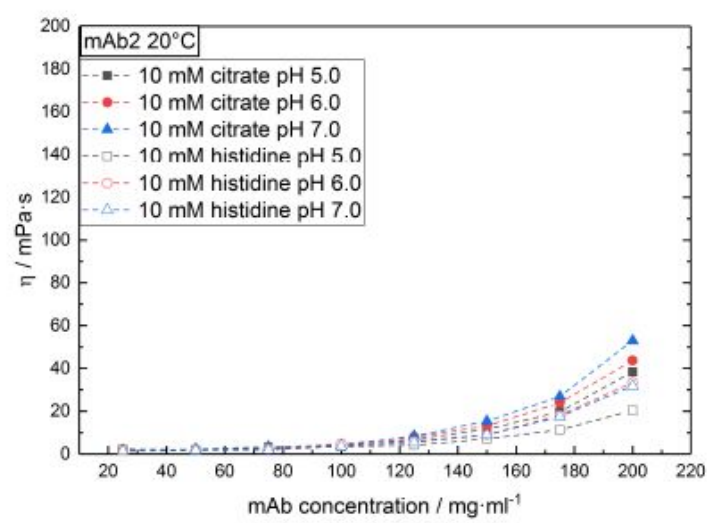

(b)

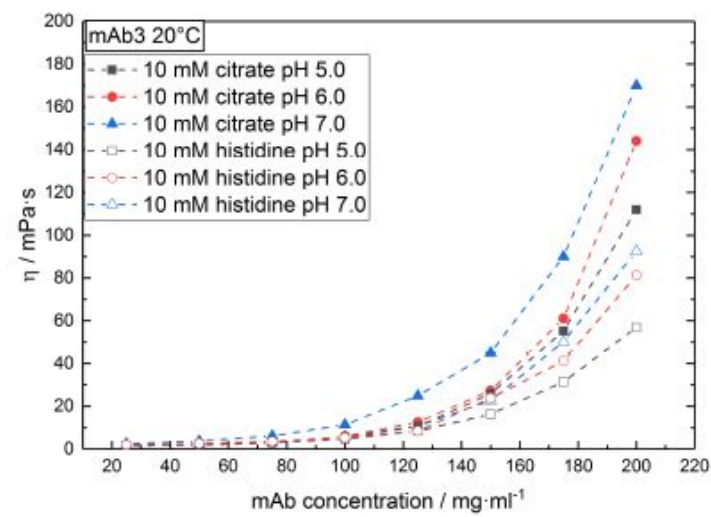

(c)

Figure S7: Dynamic viscosity in mPas of mAb solutions in different buffers at $20^{\circ} \mathrm{C}$ and shear rate 1000 $\mathrm{s}^{-1}$. Largest viscosities were found for mAb1, followed by mAb3 and mAb2. Viscosity increased with $\mathrm{pH}$ in all mAb solutions and formulations. Viscosity of mAb1 in $10 \mathrm{mM}$ citrate buffers was far higher than that of all other solutions. The dash-dotted lines in the figure are drawn to guide the eye. 